\title{
Difficult-to-Diagnose Hormone-Negative, Human Epidermal Growth Factor Receptor 2-Positive Poorly-Differentiated Breast Cancer in a Male Patient
}

\author{
Masahiro Kitada, MD*, Shunsuke Yasuda, MD, Masahiro Abe, MD, Nana Yoshida, MD, Satoshi Okazaki, \\ MD and Kei Ishibashi, MD
}

Department of Breast Disease Center, Asahikawa Medical University, Japan

\begin{abstract}
Abbreviations
HER2: Human Epithelial Growth Factor Receptor Type 2; MBC: Male Breast Cancer; CT: Computer Tomography; FDGPET: Fluorodeoxyglucose-Positron Emission Tomography; CEA: Carcinoembryonic Antigen; CA15-3: Cancer Antigen 15-3; ER: Estrogen Receptor; PgR: Progesterone Receptor; CK: Cytokeratin; GATA3: GATA Binding Protein 3; TTF-1: Thyroid Transcription Factor 1; GFDFP-15: Gross Cystic Disease RuidProtein-15; BRCA: Breast Cancer Susceptibility Gene; PARP: Poly ADP-ribose Polymerase
\end{abstract}

\section{Introduction}

Male breast cancer is rare, accounting for approximately $0.5-1.0 \%$ of all cases and is often a hormone-dependent luminal type. The percentage of hormone-negative, human epidermal growth factor receptor 2 (HER2)-positive breast cancer cases is very small in male patients (0.6-1.2\%), and the prognosis is generally poor [1-4]. We report a case of advanced hormone-negative, HER2-positive breast cancer with multiple lymph node metastases of poorly-differentiated adenocarcinoma; thus, it was difficult to identify the primary lesion.

\section{Case Report}

A 67-year-old man working in Southeast Asia presented for evaluation. The patient had visited a different hospital with general malaise and rapid weight loss. CT and MRI showed a giant mediastinal tumor and enlarged lymph nodes in the neck and thoracic cavity. The patient returned to Japan and visited our hospital. He was $165 \mathrm{~cm}$ in height and weighed $51 \mathrm{~kg}$. Edema of the upper body, including swelling of the neck was observed, and subcutaneous thickening and mass formation were observed in the left chest. Blood testing revealed a mild increase in SIL-2R to 1044 and CA15-3 to 103; the tumor markers showed no abnormalities. Computed tomography (CT) showed enlargement of the bilateral supraclavicular, mediastinal, hilar, left axillary, abdominal para-aortic, and iliac lymph nodes. The largest lesion seen was a $60-\mathrm{mm}$ tumor located in the anterior mediastinum with stenosis of the superior vena cava, suggesting malignant lymphoma (Figure 1). Positron-emission tomography showed similar findings to those observed using CT but also revealed faint uptake with a high standardized uptake value under the skin of the precordial region (Figure 2).

Based on the results of cervical lymph node biopsy, poorly-differentiated adenocarcinoma was diagnosed. Because it was difficult to identify the primary lesion, we performed biopsy of the subcutaneous thickening and mass formation on the left side of the chest. The patient was subsequently diagnosed with low-grade adenocarcinoma, similar to the initial diagnosis gleaned from the cervical lymph node biopsy. Immunostaining suggested the following: ER (-) PgR (-) HER2 (3+) (Figure 3), positive for CK7, slightly positive for GATA3 (Figure 4), negative for CK 20 and TTF-1, and negative for mammaglobin and GCDFP-15. HER2 was diagnosed as positive using the FISH test. Based on the clinical findings, the patient was finally diagnosed with T4N3M1 stage IV advanced breast cancer and scheduled the initiation of anticancer drug + anti-HER2 therapy. However, his general physical condition rapidly deteriorated, and he developed cachexia, ultimately passing away without receiving treatment 1 month after the diagnosis was made.

*Corresponding author: Masahiro Kitada, Department of Breast Disease Center, Asahikawa Medical University, MidorigaokaHigashi 2-1-1-1, Asahikawa Hokkaido 078-8510, Japan, Tel: +81166-68-2494, Fax: +81-166-68-2499

Accepted: March 10, 2020

Published online: March 12, 2020

Citation: Kitada M, Yasuda S, Abe M, et al. (2020) Difficult-toDiagnose Hormone-Negative, Human Epidermal Growth Factor Receptor 2-Positive Poorly-Differentiated Breast Cancer in a Male Patient. Ann Breast Cancer Ther 4(1):44-47 
Citation: Kitada M, Yasuda S, Abe M, et al. (2020) Difficult-to-Diagnose Hormone-Negative, Human Epidermal Growth Factor Receptor 2-Positive Poorly-Differentiated Breast Cancer in a Male Patient. Ann Breast Cancer Ther 4(1):44-47

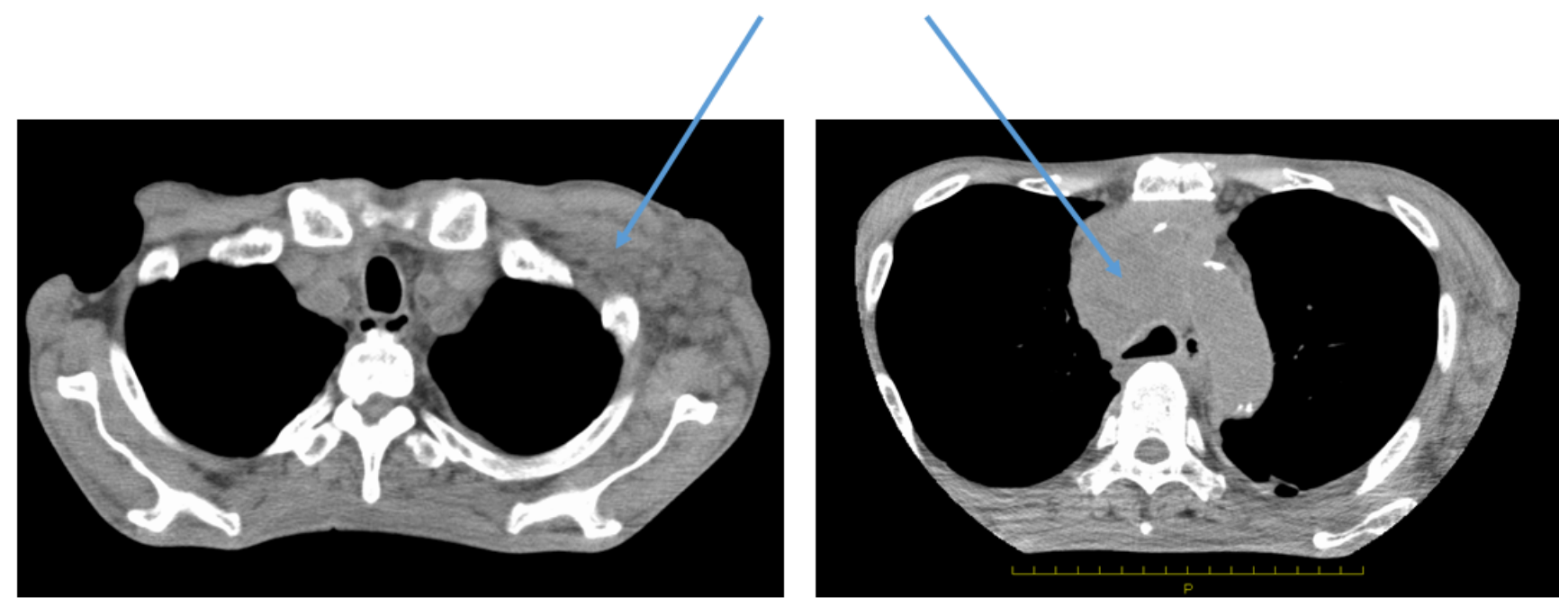

Figure 1: Chest CT shows mediastinal lymphadenopathy (arrow).
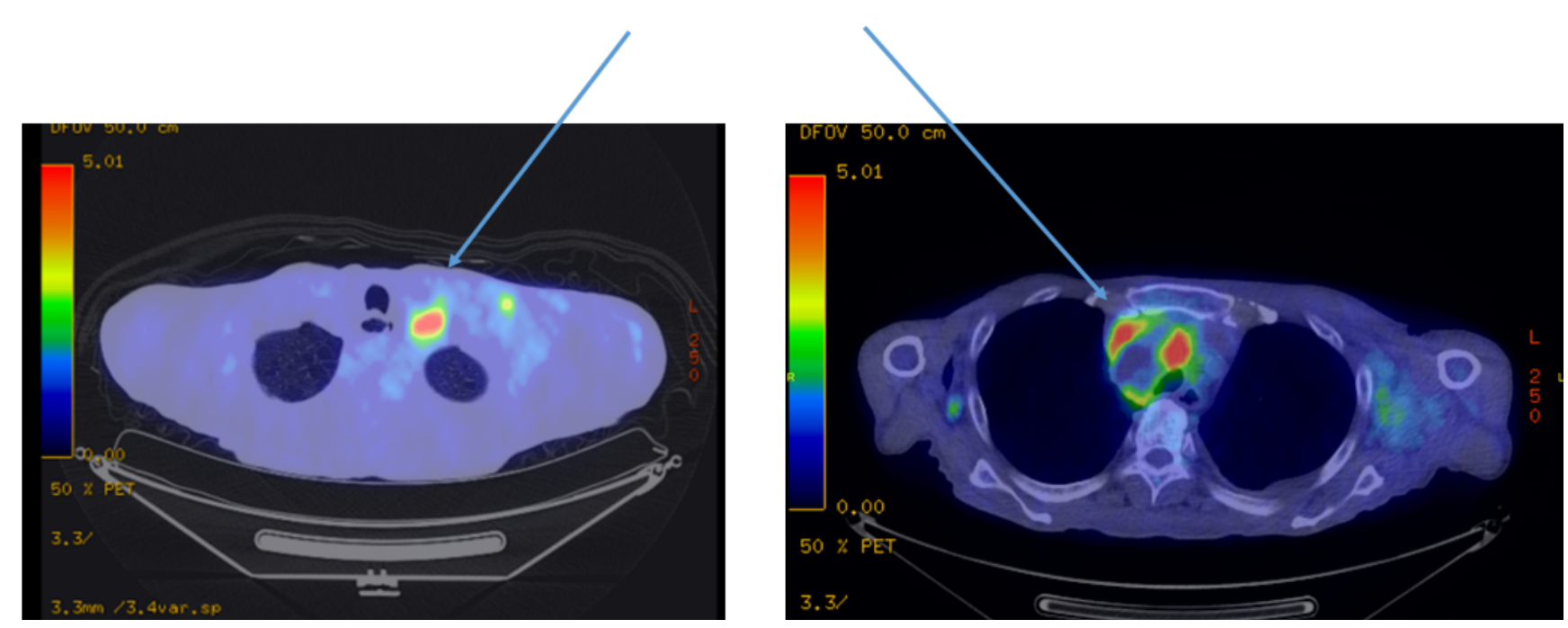

Figure 2: FDG-PET shows neck and mediastinal lymph nodes (arrows).

\section{Discussion}

Male breast cancer is rare, accounting for $0.5-1.0 \%$ of all breast cancer cases and is reportedly a hormone-dependent luminal type in many cases. The proportion of HER2 type with hormone-negative, HER2-positive MBC, as we presented herein, is as low as $0.6-1.2 \%$ among the rare MBC. It is sometimes detected in patients with advanced recurrent breast cancer, and their prognosis is often poor [1-4]. At our hospital, among 3,194 patients with breast cancer under treatment between 2000 and 2018, there were 14 (0.44\%) cases of $\mathrm{MBC}$. Of these male patients, 12 patients were hormone-dependent (ER and/or PgR positive) and HER2-negative. A total of 10 patients are still alive after 5 -years of hormone therapy only (tamoxifen), and two patients (one with stage IIIb and the other with stage IV cancer at the initial visit) died after 1-year and 8 months and 5-years and 8 months, respectively. One patient was diagnosed with hormone-negative, HER2-positive breast cancer after visiting our hospital with the chief complaints of extensive progression of right precor- dial mass and lymphoedema of the upper limbs due to multiple enlarged LN. A partial remission has been maintained with anticancer drugs and anti-HER2 therapy, and treatment is still ongoing. Together with the present case, we had an impression that HER 2 type MBC was detected in the advanced state.

We encountered a case of poorly-differentiated adenocarcinoma whose primary lesion was identified with difficulty. In addition to conducting differential diagnosis by cytokeratin staining means such as $C D 7 / 20$, it has been reported that GATA3 staining is useful in metastatic breast cancer and ER-negative breast cancer because it shows a higher positive rate than traditional breast cancer markers such as mammaglobin and GCDFP-15 [5,6]. This patient was positive for CK7 and negative for CK20 as well as negative for both GCDFP-15 and mammaglobin. Therefore, GATA3 positivity was used as an auxiliary diagnosis. GATA3 results are considered to be positive in approximately $8 \%$ of lung adenocarcinoma cases in addition to in breast cancer and urothelial carcinoma [7]. Although it was difficult to make a diagnosis in this case, the 
Citation: Kitada M, Yasuda S, Abe M, et al. (2020) Difficult-to-Diagnose Hormone-Negative, Human Epidermal Growth Factor Receptor 2-Positive Poorly-Differentiated Breast Cancer in a Male Patient. Ann Breast Cancer Ther 4(1):44-47
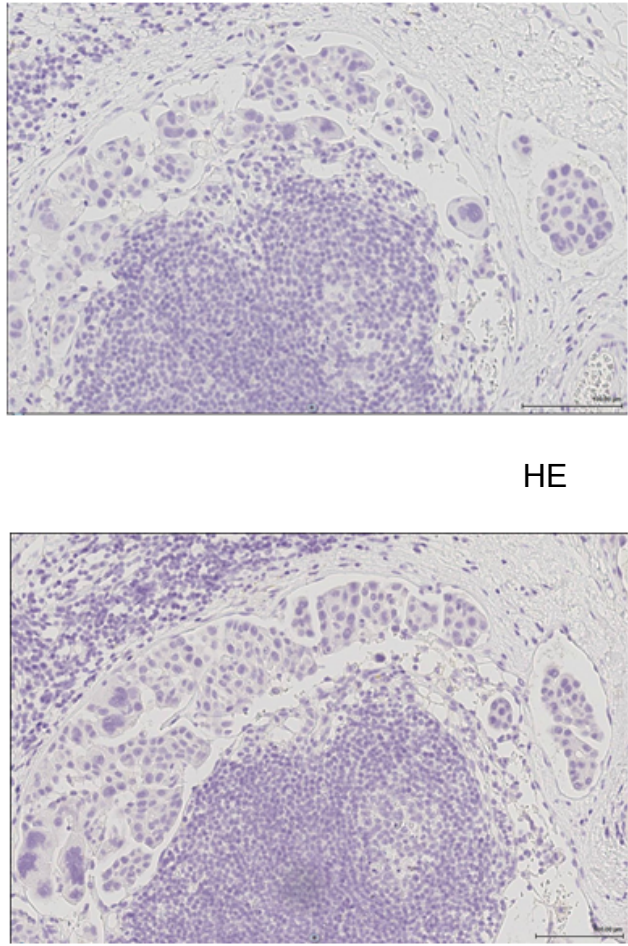

$\operatorname{PgR}$
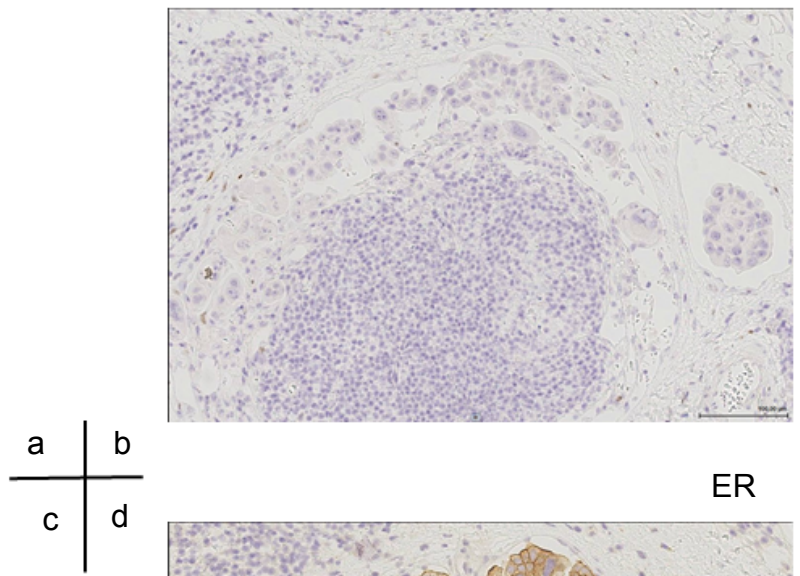

ER

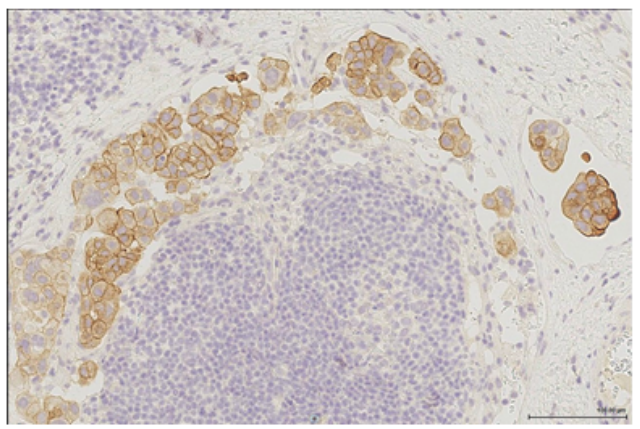

HER2

Figure 3: Immunohistochemical staining findings a) Hematoxylin-Eosin staining finding; b) Estrogen receptor staining finding; c) Progesterone receptor staining finding; d) Human Epithelial growth factor Receptor type 2 staining finding.

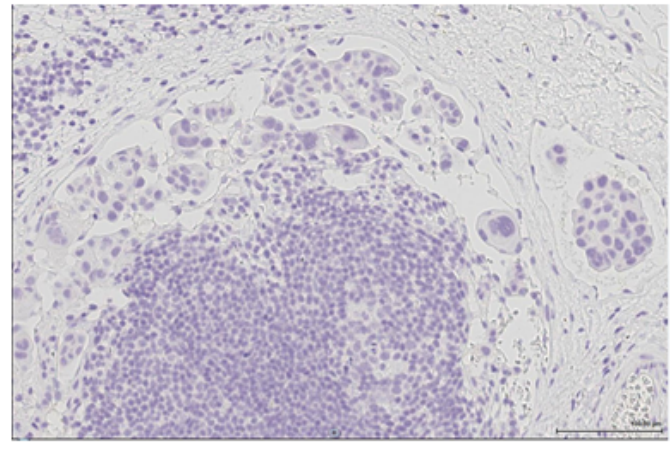

HE
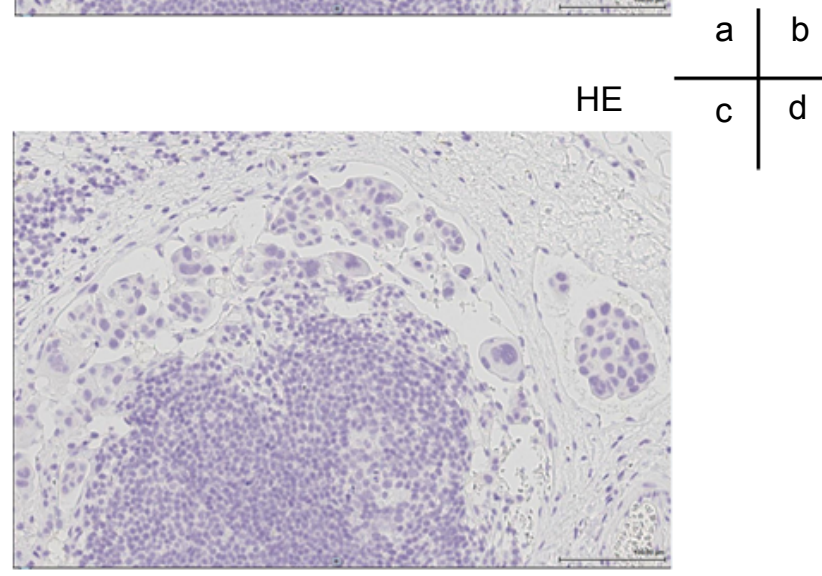

CK20

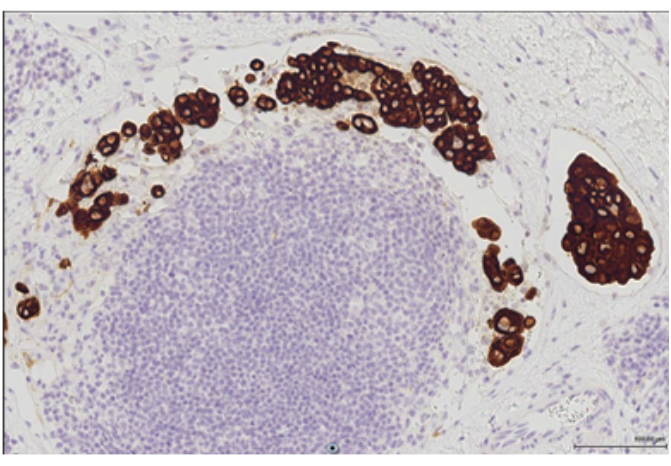

CK7

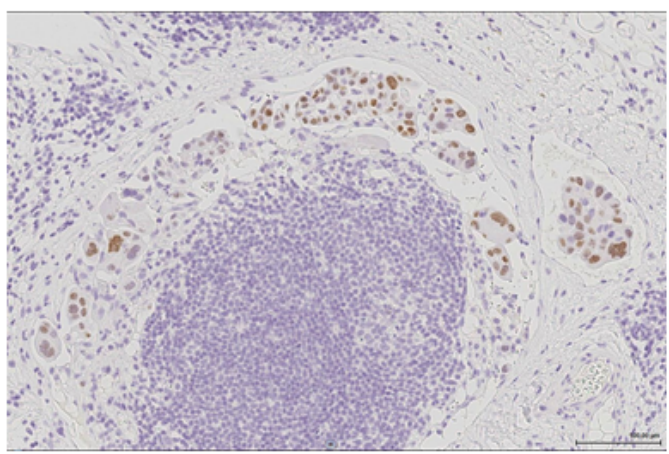

GATA3

Figure 4: Immunohistochemical staining finding a) Hematoxylin-Eosin Stain staining finding; b) Cytokeratin 7 staining finding; c) Cytokeratin 20 staining finding; d) GATA binding protein 3 staining finding. 
Citation: Kitada M, Yasuda S, Abe M, et al. (2020) Difficult-to-Diagnose Hormone-Negative, Human Epidermal Growth Factor Receptor 2-Positive Poorly-Differentiated Breast Cancer in a Male Patient. Ann Breast Cancer Ther 4(1):44-47

treatment plan for breast cancer was considered appropriate based on the biopsy site and pathological diagnosis.

Furthermore, genetic breast cancer accounts for $5-10 \%$ of breast cancer, and the proportion of BRCA mutation-positivity is reportedly higher in $\mathrm{MBC}$ than in female breast cancer $[8,9]$. BRCA mutation-positive patients are generally HER2 negative. It is believed that both HER2 loci are missing because they are close to the BRCA-1 gene. Unlike anticancer drugs, PARP inhibitors [10], which can be currently used for patients with BRCA mutation-positive advanced cases, are relatively easy to use even in poor general condition. If there are not many alternative drugs to be used like in the present case, BRCA mutation testing could have been an option for us to consider their use. In addition, in the future, exploration of treatment with gene panel tests [11] will be included as one of the study items.

\section{Conclusion}

HER2-positive breast cancer with multiple lymph node metastases of poorly-differentiated adenocarcinoma; thus, it was difficult to identify the primary lesion. GATA3 immunostaining is useful for diagnosing difficult breast cancer.

\section{Declarations}

\section{Availability of data and materials}

All data generated or analyzed during this study are included in this published article.

\section{Ethics approval and consent to participate}

All procedures used in this research were approved by the Ethics Committee of Asahikawa Medical University.

\section{Consent for publication}

Written informed consent was obtained from the patient for the publication of this case report and any accompanying images. A copy of the written consent form is available for review by the Editor-in-Chief of this journal.

\section{Competing interests}

The authors declare that they have no competing interests.

\section{Authors contribution}

MK have operated this case and analyzed all data. SY, MA, NY, SO, KI did the assistant of chemotherapy. All authors read and approved the final manuscript.

\section{Acknowledgements}

The authors declare that they have no competing interests.

\section{References}

1. Leone JP, Leone J, Zwenger AO, et al. (2015) Prognostic significance of tumor subtypes in male breast cancer: A population-based study. Breast Cancer Res Treat 152: 601-609.

2. Masci G, Caruso M, Caruso F, et al. (2015) Clinicopathological and Immunohistochemical Characteristics in Male Breast Cancer: A Retrospective Case Series. Oncologist 20: 586-592.

3. Chavez-Macgregor M, Clarke CA, Lichtensztajn D, et al. (2013) Male breast cancer according to tumor subtype and race: a population-based study. Cancer 119: 1611-1617.

4. Abreu MH, Afonso N, Abreu PH, et al. (2016) Male breast cancer: Looking for better prognostic subgroups. Breast 26: 18-24.

5. Liu H, Shi J, Prichard JW, et al. (2014) Immunohistochemical evaluation of GATA-3 expression in ER-negative breast carcinomas. Am J Clin Pathol 141: 648-655.

6. Krings $G$, Nystrom M, Mehdi I, et al. (2014) Diagnostic utility and sensitivities of GATA3 antibodies in triple-negative breast cancer. Hum Pathol 45: 2225-2232.

7. Miettinen M, McCue PA, Sarlomo-Rikala M, et al. (2014) GATA3: A multispecific but potentially useful marker in surgical pathology: A systematic analysis of 2500 epithelial and nonepithelial tumors. Am J Surg Pathol 38: 13-22.

8. Kwiatkowska E, Teresiak M, Filas V, et al. (2003) BRCA2 mutations and androgen receptor expression as independent predictors of outcome of male breast cancer patients. Clin Cancer Res 9: 4452-4459.

9. Hesse-Biber S, An C (2017) Within-gender differences in medical decision making among male carriers of the BRCA genetic mutation for hereditary breast cancer. Am J Mens Health 11: 1444-1459.

10. Tutt A, Robson M, Garber JE, et al. (2010) Oral poly (ADP-ribose) polymerase inhibitor olaparib in patients with BRCA1 or BRCA2 mutations and advanced breast cancer: A proof-of-concept trial. Lancet 376: 235-244.

11. Zehir A, Benayed R, Shah RH, et al. (2017) Mutational landscape of metastatic cancer revealed from prospective clinical sequencing of 10,000 patients. Nat Med 23: 703-713.

DOI: $10.36959 / 739 / 524$

Copyright: (c) 2020 Kitada M, et al. This is an open-access article distributed under the terms of the Creative Commons Attribution License, which permits unrestricted use, distribution, and reproduction in any medium, provided the original author and source are credited. 